\title{
Analysis of Problematic Smartphone Use Across Different Age Groups within the 'Components Model of Addiction'
}

\author{
Sándor Csibi ${ }^{1} \cdot$ Mark D. Griffiths $^{2}$ (D) $\cdot$ Zsolt Demetrovics $^{3} \cdot$ Attila Szabo $^{3,4}$ \\ Published online: 23 May 2019 \\ (C) The Author(s) 2019
}

\begin{abstract}
Smartphones have rapidly become indispensable in modern life. The wide spectrum of activities and virtual interaction possibilities with the mobile device render some individuals prone to exaggerated use exhibiting core symptoms akin to substance addiction. The core common symptoms of addictions can be framed within the 'components model of addiction.' Based on the different pattern of smartphone use in various age groups, the symptoms and the risk of smartphone addiction may differ during various life stages. Some age groups may be more at risk of smartphone addiction than others. This hypothesis was tested utilizing data from six surveys comprising 1603 individuals representing various age groups. Results obtained through two recommended age-group analyses both confirmed that preschool children and young adults are at highest risk for smartphone-related addictive behavior. Furthermore, the various components of addiction also differed between the age groups. These results suggest that preventive measures should be primarily targeted to the two most vulnerable age groups represented by preschool children and emerging adults. The results of the present study help identify the dominant addiction symptoms in the various age groups that could pinpoint aspects which should be targeted in implementation of various measures aimed at the prevention of dysfunctional smartphone use.
\end{abstract}

Keywords Smartphone use · Age groups · 'Components model of addiction' · Problematic smartphone use $\cdot$ Smartphone addiction

Mark D. Griffiths

mark.griffiths@ntu.ac.uk

1 Department of Ethics and Social Sciences, University of Medicine and Pharmacy, Târgu Mureş, Romania

2 Behavioural Addiction, Psychology Department, Nottingham Trent University, 50 Shakespeare Street, Nottingham NG14FQ, UK

3 Institute of Psychology, ELTE Eötvös Loránd University, Budapest, Hungary

4 Institute of Health Promotion and Sport Sciences, ELTE Eötvös Loránd University, Budapest, Hungary 
From a developmental psychological perspective, research on smartphone use in the literature mainly focuses on young adults, so the most commonly studied samples are typically university students. The most vulnerable age group from the point of view of poor mental health and excessive smartphone use are adolescents aged 14 to 18 years (Lemola et al. 2015). However, younger children also appear to suffer from problematic smartphone use (Park and Park 2014). The UK Office of Communications (Ofcom) reported that 8- to 11-year-old smartphone users spend $10 \mathrm{~h}$ a week (on average) on their smartphone, which is less than the time spent by 10- to 12-year-olds (spending just over $18 \mathrm{~h}$ ) (Ofcom 2017). According to statistics by age group, children's smartphone usage is 35\% among those aged 3-4 years, 38\% for 5-7 years, $75 \%$ for 8-11 years, and $86 \%$ for 12-15 years (Ofcom 2017). Since smartphone use may begin as early as 2 years of age (Kim and Kang, 2016), analyses have recently been extended to younger age groups of children aged between 6 and 10 years, and even among groups of preschoolers (Yadav and Chakraborty 2017).

Problematic smartphone use can be viewed from the perspective of the 'components model of addiction' (Griffiths, 2005), which posits that addictive behavior can be identified by the presence of six core components. In the context of smartphone use, these are the overwhelming dominance and total preoccupation of smartphone use (salience), mood changes experienced directly through the use of smartphones (mood modification), the increase over time of daily hours spent using smartphones (tolerance), negative feelings when unable to engage in smartphone use (withdrawal symptoms), intrapersonal and interpersonal problems arising from smartphone use (conflict), and the reversion to addictive smartphone behavior after a period of abstinence (relapse). Based on the addiction components model, many psychometric scales have now been developed to assess the presence of different behavioral addictions including exercise addiction (Terry et al. 2004), work addiction (Andreassen et al. 2012), social media addiction (Andreassen et al. 2016), shopping addiction (Andreassen et al. 2015), and problematic pornography use (Böthe et al. 2018).

To assess the problematic use of smartphone applications, a new scale was recently developed - the Brief Addiction to Smartphone Scale (BASS; Csibi et al. 2016), and was psychometrically validated for the Hungarian population, being used to indicate the presence of psychological and behavioral problems related to excessive smartphone use. The Englishlanguage adult version of the BASS, the SABAS (Smartphone Application-Based Addiction Scale [Csibi et al. 2018]) was validated using English-speaking populations. Based on psychometric testing, the SABAS is a brief, valid, and reliable instrument that quickly and easily assesses application-based addiction symptoms arising from smartphone use. Across the human life span, people of different ages engage in activities specific to their age group. Games, peer groups, education, work, sexual relationships, family responsibilities, and retirement all affect daily schedules and lifestyle. The use of the smartphone, thanks to its various functions, is part of modern lifestyle irrespective of age. In short, age affects individuals' way of life. Because lifestyle differs depending upon age, everyday habits (such as smartphone use) should be analyzed by considering age characteristics (Kuss et al. 2018). For example, the evolving social identity of teens are moderated by feedback from their peers, often based on the speed of responses to messages, the level and type of use of a smartphone, and the online permanency, all these leading to the desired peer-status and gaining popularity among younger users (Fullwood et al. 2017). Furthermore, for developing self-control in childhood, the continuous signals from their smartphones are strongly challenging, by choosing between the urge to respond or to delay the response in favor of a current activity (Berger et al. 2018; Ihm 2018). 
The research aims of the present study were to address the following questions: (i) do problematic symptoms in the components model (salience, mood modification, tolerance, withdrawal symptoms, conflict, and relapse) show different patterns among age groups and developmental stages? and (ii) are there age groups at greater risk for developing smartphone applicationbased addictions? Furthermore, it was hypothesized that the risk of smartphone application-based addictions would differ between various age groups, with younger people being more affected.

\section{Methods}

\section{Sample}

The study sample and sampling procedures varied across different age groups. The data were collated from six different surveys (of previously published and unpublished data). This multiple-sampling approach resulted in a total of 1651 participants across different age groups who completed the SABAS. After eliminating responses from participants with incomplete data, the final number of participants was 1603 (Fig. 1).

In the kindergarten, a short parental questionnaire was used, involving interviewers to collect children' individual answers. This was a time-consuming procedure and resulted in 75 participants (mean age $=6.01$ years, $\mathrm{SD}=1.4$ ) (unpublished data). Thus, the total sample included elementary school-aged children sample $(n=258$, mean age $=12.4$ years, SD $=1.7)($ Csibi et al., 2017) and high school student sample $(n=441$, mean age $=13.4$ years, $\mathrm{SD}=2.2)$, where the data were collected via paper-based scales completion, applied in school classes (Csibi et al. 2016). Adults $(n=589)$ and older adults $(n=69)$ were invited to participate in an online study using the Qualtrics software via adverts on different social media. These data stem from three surveys, the first contained 240 respondents (mean age $=25.4$ years, $\mathrm{SD}=8.1$; Csibi et al. 2018), the second 442 respondents (mean age $=28,5, \mathrm{SD}=12.7$; Csibi et al. 2019), and the third 147 respondents (mean age $=37.6$ years, $\mathrm{SD}=13.4$; previously unpublished data).

\section{Instruments}

Problematic smartphone use was assessed using the (i) Hungarian version of the SABAS (BASS), which is a valid and reliable tool for assessing applications-based problematic

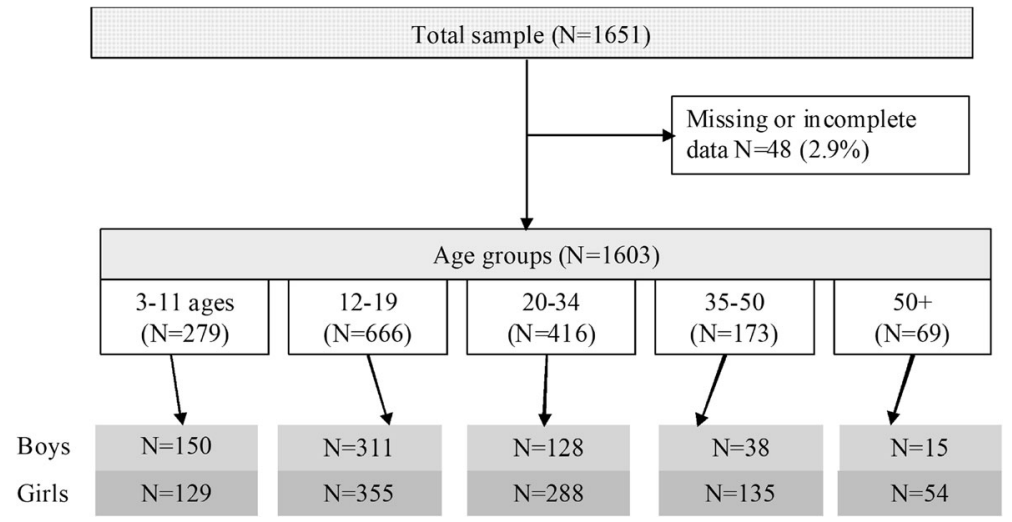

Fig. 1 Diagram of age and gender distribution of the sample 
smartphone use among kindergarten children (under validation), schoolchildren (Csibi et al. 2016), and adults (Csibi et al. 2019) and (ii) English version of the SABAS validated in an international adult population (Csibi et al. 2018). The principal components analysis of the SABAS (BASS) reported a single component for the six items, which accounted for $53 \%$ of the total variance in the school children' sample, and $35 \%$ of the variance in adults' sample with Hungarian participants. The internal reliability of the scale was good for schoolchildren (Cronbach's alpha $=0.82$; Csibi et al. 2016) and for adults (Cronbach's alpha $=0.75$; Csibi et al. 2019). The English version of the scale also showed one component, which accounted for $52 \%$ of the total variance (Cronbach's alpha 0.81; Csibi et al. 2018).

\section{Procedure}

To assess age-group differences, the stage of life classification system was used (Armstrong 2007). This groups individuals into childhood (3-11 years), adolescence (12-19 years), young adulthood (20-34 years), adulthood (35-50 years), and older adulthood (51+ years). However, smartphone use can be determined by the individual's most time-consuming daily activities. Accordingly, the division of age groups can be adapted to specific, everyday activities. Therefore, for a more detailed description of the results, a different, more accurate classification was also used (as recommended by Kail and Cavanaugh 2018). This division is based on the following age grouping: (i) mostly kindergarten, (ii) generally elementary school, (iii) generally high school, (iv) mainly professional or graduate school, (v) early adulthood, (vi) adulthood, and (vii) middle adulthood and above.

\section{Statistical Analysis}

A multivariate analysis of variance (MANOVA), on the six components of the SABAS with age and gender being the grouping factors, was used to test the hypothesis. The statistics were performed using Statistical Package for Social Science (SPSS software, version 21 [IBM Inc]).

\section{Results}

The problematic smartphone use total score analysis showed a significant effect utilizing Armstrong's age group classification on SABAS total scores $(F[4,1597]=25.179, p<0.001)$. According to the more detailed analysis of the age groups, the overall score of those aged 3-11 years was significantly different from those aged 12-19 years and 20-34 years. There were also significant differences between those aged 12-19 years and those aged 20-34 years, as well as differences between those aged 20-34 years and those aged 35-50 years and those over 50 years old (Table 1).

A graphical representation of the findings showed that the 20-34-year age group had the highest score on the problematic smartphone usage scale, followed by those aged 3-11 years and those aged 35-50 years. The lowest scores were for those aged 11-19 years and those over 50 years of age (Fig. 2).

Analysis of the problematic smartphone use-related components showed relevant differences by age group, and covariance with gender (Table 2). There was a significant effect of age groups on all problematic use components (Pillai's trace $=0.297 ; F(24,6380)=21.35$, $p<0.001)$. The analysis was repeated for each factor by age group. Results showed differences for each age group for each factor of problematic smartphone use (Table 3 ). 
Table 1 Means, standard deviation, and distribution of Smartphone Application-Based Addiction Scale score by age and gender ( $\mathrm{M}=$ male; $\mathrm{F}=$ female) by Armstrong's age groups classification

\begin{tabular}{|c|c|c|c|c|c|c|c|c|c|}
\hline \multirow[t]{2}{*}{ Age group } & \multirow[t]{2}{*}{ Number } & \multicolumn{6}{|c|}{ BASS items (mean, SD) } & \multirow{2}{*}{$\begin{array}{l}\text { SABAS total score } \\
\text { (mean, SD) }\end{array}$} & \multirow{2}{*}{$\begin{array}{l}\text { Sign. }^{\mathrm{a}, \mathrm{b}} \\
p\end{array}$} \\
\hline & & 1 & 2 & 3 & 4 & 5 & 6 & & \\
\hline $3-11$ years & 279 & $\begin{array}{l}3.18 \\
(1.76)\end{array}$ & $\begin{array}{l}2.73 \\
(2.08)\end{array}$ & $\begin{array}{l}3.80 \\
(1.76)\end{array}$ & $\begin{array}{l}3.15 \\
(1.65)\end{array}$ & $\begin{array}{l}2.57 \\
(1.72)\end{array}$ & $\begin{array}{l}2.39 \\
(1.64)\end{array}$ & $\begin{array}{l}17.80 \\
(7.02)\end{array}$ & $\begin{array}{l}0.005^{1} \\
<0.001^{2}\end{array}$ \\
\hline $12-19$ years & 666 & $\begin{array}{l}2.65 \\
(1.42)\end{array}$ & $\begin{array}{l}2.46 \\
(1.62)\end{array}$ & $\begin{array}{l}3.32 \\
(1.54)\end{array}$ & $\begin{array}{l}2.84 \\
(1.51)\end{array}$ & $\begin{array}{l}2.51 \\
(1.56)\end{array}$ & $\begin{array}{l}2.42 \\
(1.45)\end{array}$ & $\begin{array}{l}16.19 \\
(6.41)\end{array}$ & $<0.001^{2}$ \\
\hline 20-34 years & 416 & $\begin{array}{l}3.56 \\
(1.36)\end{array}$ & $\begin{array}{l}3.35 \\
(1.64)\end{array}$ & $\begin{array}{l}3.23 \\
(1.29)\end{array}$ & $\begin{array}{l}2.70 \\
(1.31)\end{array}$ & $\begin{array}{l}3.93 \\
(1.33)\end{array}$ & $\begin{array}{l}3.24 \\
(1.50)\end{array}$ & $\begin{array}{l}20.00 \\
(5.91)\end{array}$ & $<0.001^{3,4}$ \\
\hline $35-50$ years & 173 & $\begin{array}{l}3.27 \\
(1.42)\end{array}$ & $\begin{array}{l}2.51 \\
(1.47)\end{array}$ & $\begin{array}{l}2.71 \\
(1.32)\end{array}$ & $\begin{array}{l}2.39 \\
(1.29)\end{array}$ & $\begin{array}{l}3.31 \\
(1.41)\end{array}$ & $\begin{array}{l}2.53 \\
(1.40)\end{array}$ & $\begin{array}{l}16.72 \\
(6.25)\end{array}$ & \\
\hline $50+$ years & 69 & $\begin{array}{l}3.45 \\
(1.44)\end{array}$ & $\begin{array}{l}2.25 \\
(1.45)\end{array}$ & $\begin{array}{l}2.58 \\
(1.44)\end{array}$ & $\begin{array}{l}2.29 \\
(1.31)\end{array}$ & $\begin{array}{l}3.20 \\
(1.44)\end{array}$ & $\begin{array}{l}2.43 \\
(1.32)\end{array}$ & $\begin{array}{l}16.20 \\
(6.33)\end{array}$ & \\
\hline Total N & 1603 & $\begin{array}{l}3.08 \\
(1.52)\end{array}$ & $\begin{array}{l}2.73 \\
(1.73)\end{array}$ & $\begin{array}{l}3.28 \\
(1.53)\end{array}$ & $\begin{array}{l}2.78 \\
(1.47)\end{array}$ & $\begin{array}{l}3.01 \\
(1.63)\end{array}$ & $\begin{array}{l}2.64 \\
(1.53)\end{array}$ & $\begin{array}{l}17.52 \\
(6.56)\end{array}$ & \\
\hline
\end{tabular}

a The mean difference is significant at the 0.05 level

b Adjustment for multiple comparisons: Bonferroni

${ }^{1}$ Compared to the $12-19$ age group

${ }^{2}$ Compared to the 20-35 age group

${ }^{3}$ Compared to the $35-50$ age group

${ }^{4}$ Compared to the $50+$ age group

Table 3 demonstrates that most significant differences were found in the components of mood modification and withdrawal symptoms, where the results of almost all age groups differed significantly. Thus, the group aged 3-11 years had the highest values for salience, mood modification, and tolerance, and the group aged 12-19 years had the highest values for mood modification and tolerance. The highest scores of the 20-34-year age group were for salience, conflict, withdrawal symptoms, and relapse factors. The highest scores of the 35-50year age group were for salience and withdrawal symptoms, whereas it was salience for the over 50 years age group.

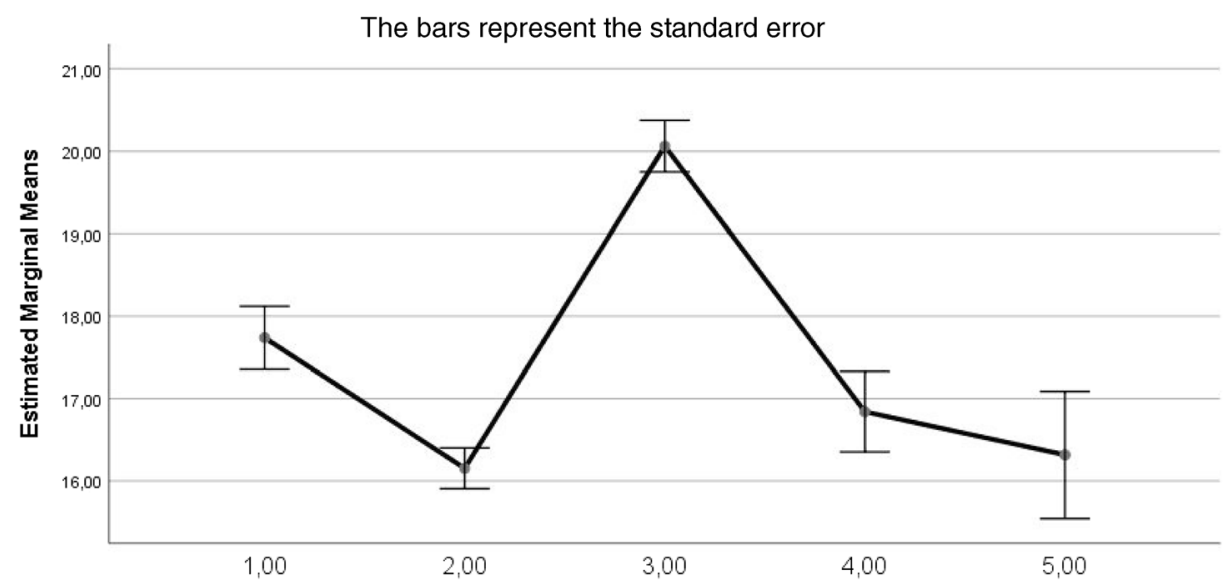

Fig. 2 SABAS total score variation by Armstrong's age groups 
Table 2 Tests of between-participant effects and significance of the problematic use components by Armstrong's age groups

\begin{tabular}{llllllll}
\hline Source & Dependent variable & $\begin{array}{l}\text { Type III sum } \\
\text { of square }\end{array}$ & df. & $\begin{array}{l}\text { Mean } \\
\text { square }\end{array}$ & $F$ & Sig.* & $\begin{array}{l}\text { Partial Eta } \\
\text { squared }\end{array}$ \\
\hline Age group & Salience & 228.66 & 4 & 57.16 & 26.23 & $p<0.001$ & 0.0622 \\
& Conflict & 237.82 & 4 & 59.45 & 20.71 & $p<0.001$ & 0.049 \\
& Mood modification & 146.51 & 4 & 36.62 & 16.33 & $p<0.001$ & 0.039 \\
& Tolerance & 73.11 & 4 & 18.27 & 8.61 & $p<0.001$ & 0.021 \\
& Withdrawal symptoms & 578.91 & 4 & 144.72 & 62.82 & $p<0.001$ & 0.136 \\
& Relapse & 209.17 & 4 & 52.29 & 23.54 & $p<0.001$ & 0.056 \\
& SABAS total score & 4092.51 & 4 & 1023.12 & 25.17 & $p<0.001$ & 0.059 \\
\hline
\end{tabular}

*Computed using alpha $=0.05$

Most behaviors develop around an individual's day-to-day activities. These include school time for schoolchildren, courses schedule for university students, and a daily program defined by the workplace for adults. Accordingly, smartphone use may differ within some of those age groups, especially among younger people. Therefore, in addition to Armstrong's age group division, it was necessary to examine in more detail the characteristics of the smartphone usage of the respondents. The results were also examined by another age group (Kail and Cavanaugh 2018), which also considered the typical activities of the given age (Table 4). The problematic smartphone use total score analysis showed a significant effect of Kail and Cavanaugh's age group classification on SABAS total scores $(F[61590]=187.109, p<0.001)$ (Fig. 3).

Analysis of the problematic smartphone use-related components showed relevant differences by age group, and covariance with gender (Table 5). There was a significant effect of age groups on all problematic use components (Pillai's trace $=.425 ; F(36,9570)=20.24$, $p<0.001$ ). Therefore, the analysis was repeated for each factor by age group. Results showed differences for each age group for each factor of problematic smartphone usage (Table 6).

According to the results, data of different age groups utilizing Kail and Cavanaugh's age groups classification, showed significantly different averages. The results for the 3-6-year-olds were significantly different from those of any other age group, especially for the relapse component, while the results of 7-11 year olds were significantly different from those of all other age groups for the tolerance component.

\section{Discussion}

Smartphone use is widespread in industrialized societies and discernible across all age groups. Based on the findings of the present study, the extent and characteristics of problematic smartphone use appear to show important differences depending upon age. More specifically, the results of the present study show that problematic use of smartphones is age-dependent, since each age group had significantly different scores on the problematic smartphone use scale. Other research in the field shows that age is a predictor of problematic smartphone use risk, especially in the case of 16-25-year and 26-35-year old groups (de-Sola et al. 2017; Kwon et al. 2017). According to the results, the 20-34-year old age group reported the highest scores on the BASS/SABAS, followed by the 3-11-year olds. Based on two analyses, contrary to expectations, the lowest scores were found among participants aged between 12 and 19 years. The oldest age group exhibited the lowest BASS/SABAS scores from the adult 
Table 3 Multiple comparison results for the problematic use components by Armstrong's age groups

\begin{tabular}{|c|c|c|c|c|c|}
\hline \multirow{2}{*}{$\begin{array}{l}\text { Dependent variable } \\
\text { Salience }\end{array}$} & \multicolumn{2}{|c|}{ Age group (years) } & \multirow{2}{*}{$\frac{\text { Mean difference }(\mathrm{I}-\mathrm{J})}{0.521^{*}}$} & \multirow{2}{*}{$\frac{\text { Std. error }}{0.105}$} & \multirow{2}{*}{$\frac{\text { Sig.b }}{p<0.001}$} \\
\hline & $3-11$ & $12-19$ & & & \\
\hline & & $20-34$ & $-0.383^{*}$ & 0.115 & 0.009 \\
\hline & & $35-50$ & -0.092 & 0.145 & 1.000 \\
\hline & & $50+$ & -0.276 & 0.200 & 1.000 \\
\hline & $12-19$ & $20-34$ & $-0.904^{*}$ & 0.093 & $p<0.001$ \\
\hline & & $35-50$ & $-0.609^{*}$ & 0.127 & $p<0.001$ \\
\hline & & $50+$ & $-0.793^{*}$ & 0.188 & $p<0.001$ \\
\hline & $20-34$ & $35-50$ & 0.291 & 0.134 & 0.296 \\
\hline & & $50+$ & 0.108 & 0.192 & 1.000 \\
\hline & $35-50$ & $50+$ & -0.183 & 0.210 & 1.000 \\
\hline \multirow[t]{10}{*}{ Conflict } & $3-11$ & $12-19$ & 0.259 & 0.121 & 0.323 \\
\hline & & $20-34$ & $-0.655^{*}$ & 0.132 & $p<0.001$ \\
\hline & & $35-50$ & 0.163 & 0.166 & 1.000 \\
\hline & & $50+$ & 0.431 & 0.229 & 0.602 \\
\hline & $12-19$ & $20-34$ & $-0.914^{*}$ & 0.107 & $p<0.001$ \\
\hline & & $35-50$ & -0.096 & 0.146 & 1.000 \\
\hline & & $50+$ & 0.172 & 0.215 & 1.000 \\
\hline & $20-34$ & $35-50$ & $0.819^{*}$ & 0.153 & $p<0.001$ \\
\hline & & $50+$ & $1.086^{*}$ & 0.220 & $p<0.001$ \\
\hline & $35-50$ & $50+$ & 0.268 & 0.241 & 1.000 \\
\hline \multirow[t]{10}{*}{ Mood modification } & $3-11$ & $12-19$ & $0.473^{*}$ & 0.107 & $p<0.001$ \\
\hline & & $20-34$ & $0.534^{*}$ & 0.117 & $p<0.001$ \\
\hline & & $35-50$ & $1.036^{*}$ & 0.147 & $p<0.001$ \\
\hline & & $50+$ & $1.167^{*}$ & 0.203 & $p<0.001$ \\
\hline & $12-19$ & $20-34$ & 0.061 & 0.094 & 1.000 \\
\hline & & $35-50$ & $0.563^{*}$ & 0.129 & $p<0.001$ \\
\hline & & $50+$ & $0.694^{*}$ & 0.190 & 0.003 \\
\hline & $20-34$ & $35-50$ & $0.502^{*}$ & 0.136 & 0.002 \\
\hline & & $50+$ & $0.633^{*}$ & 0.195 & 0.012 \\
\hline & $35-50$ & $50+$ & 0.131 & 0.213 & 1.000 \\
\hline \multirow[t]{10}{*}{ Tolerance } & 3-11 & $12-19$ & $0.306^{*}$ & 0.104 & 0.033 \\
\hline & & $20-34$ & $0.419^{*}$ & 0.114 & 0.002 \\
\hline & & $35-50$ & $0.716^{*}$ & 0.143 & $p<0.001$ \\
\hline & & $50+$ & $0.819^{*}$ & 0.197 & $p<0.001$ \\
\hline & $12-19$ & $20-34$ & 0.113 & 0.092 & 1.000 \\
\hline & & $35-50$ & $0.410^{*}$ & 0.126 & 0.011 \\
\hline & & $50+$ & 0.513 & 0.185 & 0.057 \\
\hline & $20-34$ & $35-50$ & 0.297 & 0.132 & 0.246 \\
\hline & & $50+$ & 0.400 & 0.190 & 0.350 \\
\hline & $35-50$ & $50+$ & 0.103 & 0.207 & 1.000 \\
\hline \multirow[t]{10}{*}{ Withdrawal symptoms } & $3-11$ & $12-19$ & 0.060 & 0.108 & 1.000 \\
\hline & & $20-34$ & $-1.366^{*}$ & 0.118 & $p<0.001$ \\
\hline & & $35-50$ & $-0.747^{*}$ & 0.149 & $p<0.001$ \\
\hline & & $50+$ & $-0.638^{*}$ & 0.205 & 0.019 \\
\hline & 12-19 & $20-34$ & $-1.427^{*}$ & 0.096 & $p<0.001$ \\
\hline & & $35-50$ & $-0.808^{*}$ & 0.131 & $p<0.001$ \\
\hline & & $50+$ & $-0.698^{*}$ & 0.193 & 0.003 \\
\hline & $20-34$ & $35-50$ & $0.619^{*}$ & 0.137 & $p<0.001$ \\
\hline & & $50+$ & $0.728^{*}$ & 0.197 & 0.002 \\
\hline & $35-50$ & $50+$ & 0.109 & 0.216 & 1.000 \\
\hline \multirow[t]{5}{*}{ Relapse } & $3-11$ & $12-19$ & -0.034 & 0.106 & 1.000 \\
\hline & & $20-34$ & $-0.874^{*}$ & 0.116 & $p<0.001$ \\
\hline & & $35-50$ & -0.179 & 0.146 & 1.000 \\
\hline & & $50+$ & -0.083 & 0.202 & 1.000 \\
\hline & $12-19$ & $20-34$ & $-0.839^{*}$ & 0.094 & $p<0.001$ \\
\hline
\end{tabular}


Table 3 (continued)

\begin{tabular}{|c|c|c|c|c|c|}
\hline \multirow[t]{6}{*}{ Dependent variable } & \multicolumn{2}{|c|}{ Age group (years) } & \multirow{2}{*}{$\begin{array}{l}\text { Mean difference (I-J) } \\
-0.145\end{array}$} & \multirow{2}{*}{$\frac{\text { Std. error }}{0.129}$} & \multirow{2}{*}{$\frac{\text { Sig. }{ }^{b}}{1.000}$} \\
\hline & & $35-50$ & & & \\
\hline & & $50+$ & -0.048 & 0.189 & 1.000 \\
\hline & $20-34$ & $35-50$ & $0.694^{*}$ & 0.135 & $p<0.001$ \\
\hline & & $50+$ & $0.791^{*}$ & 0.194 & $p<0.001$ \\
\hline & $35-50$ & $50+$ & 0.097 & 0.212 & 1.000 \\
\hline
\end{tabular}

*The mean difference is significant at the 0.05 level

b Adjustment for multiple comparisons: Bonferroni

participants. These findings are in line with the study of Van Deursen et al. (2015) suggesting that age and smartphone use show an inverse relation, so that as individuals get older, the less time they spend using their smartphone for social interaction. Consequently, they are less likely to develop habitual or addictive behaviors related to the smartphone (Van Deursen et al. 2015).

However, according to Lane and Manner (2011), older individuals with higher education are more likely to use smartphones. Given the present study did not collect data concerning education level, possible differences in smartphone use between more or less-educated older

Table 4 Means, standard deviation, and distribution of Smartphone Application-Based Addiction Scale score by age and gender $(\mathrm{M}=$ male; $\mathrm{F}=$ female $)$ by Kail and Cavanaugh's age groups classification

\begin{tabular}{|c|c|c|c|c|c|c|c|c|c|}
\hline \multirow[t]{2}{*}{ Age group } & \multirow[t]{2}{*}{ Number } & \multicolumn{6}{|c|}{ SABAS items (mean, SD) } & \multirow{2}{*}{$\begin{array}{l}\text { SABAS total score } \\
\text { (mean, SD) }\end{array}$} & \multirow{2}{*}{$\begin{array}{l}\text { Sign. }{ }^{\mathrm{a}, \mathrm{b}} \\
p\end{array}$} \\
\hline & & 1 & 2 & 3 & 4 & 5 & 6 & & \\
\hline $3-6$ years & 51 & $\begin{array}{l}3.88 \\
(1.87)\end{array}$ & $\begin{array}{l}3.843 \\
(2.33)\end{array}$ & $\begin{array}{l}3.80 \\
(1.62)\end{array}$ & $\begin{array}{l}2.45 \\
(1.41)\end{array}$ & $\begin{array}{l}2.22 \\
(1.52)\end{array}$ & $\begin{array}{l}1.67 \\
(.93)\end{array}$ & $\begin{array}{l}17.86 \\
(5.37)\end{array}$ & \\
\hline $7-11$ years & 228 & $\begin{array}{l}3.02 \\
(1.70)\end{array}$ & $\begin{array}{l}2.48 \\
(1.94)\end{array}$ & $\begin{array}{l}3.79 \\
(1.79)\end{array}$ & $\begin{array}{l}3.30 \\
(1.66)\end{array}$ & $\begin{array}{l}2.65 \\
(1.76)\end{array}$ & $\begin{array}{l}2.55 \\
(1.72)\end{array}$ & $\begin{array}{l}17.79 \\
(7.35)\end{array}$ & $\begin{array}{l}<0.001^{1} \\
0.001^{2}\end{array}$ \\
\hline $12-18$ years & 555 & $\begin{array}{l}2.48 \\
(1.39)\end{array}$ & $\begin{array}{l}2.35 \\
(1.61)\end{array}$ & $\begin{array}{l}3.34 \\
(1.59)\end{array}$ & $\begin{array}{l}2.88 \\
(1.53)\end{array}$ & $\begin{array}{l}2.30 \\
(1.52)\end{array}$ & $\begin{array}{l}2.34 \\
(1.46)\end{array}$ & $\begin{array}{l}15.67 \\
(6.51)\end{array}$ & $\begin{array}{l}<0.001^{2} \\
<0.001^{3}\end{array}$ \\
\hline 19-24 years & 400 & $\begin{array}{l}3.54 \\
(1.31)\end{array}$ & $\begin{array}{l}3.34 \\
(1.59)\end{array}$ & $\begin{array}{l}3.22 \\
(1.25)\end{array}$ & $\begin{array}{l}2.73 \\
(1.32)\end{array}$ & $\begin{array}{l}3.89 \\
(1.32)\end{array}$ & $\begin{array}{l}3.17 \\
(1.47)\end{array}$ & $\begin{array}{l}19.89 \\
(5.64)\end{array}$ & $\begin{array}{l}<0.001^{4} \\
<0.001^{5}\end{array}$ \\
\hline $25-34$ & 127 & $\begin{array}{l}3.58 \\
(1.46)\end{array}$ & $\begin{array}{l}3.09 \\
(1.69)\end{array}$ & $\begin{array}{l}3.26 \\
(1.40)\end{array}$ & $\begin{array}{l}2.55 \\
(1.30)\end{array}$ & $\begin{array}{l}3.73 \\
(1.42)\end{array}$ & $\begin{array}{l}3.11 \\
(1.49)\end{array}$ & $\begin{array}{l}19.33 \\
(6.17)\end{array}$ & $<0.001^{5}$ \\
\hline $35-44$ & 132 & $\begin{array}{l}3.33 \\
(1.41)\end{array}$ & $\begin{array}{l}2.64 \\
(1.54)\end{array}$ & $\begin{array}{l}2.77 \\
(1.31)\end{array}$ & $\begin{array}{l}2.45 \\
(1.31)\end{array}$ & $\begin{array}{l}3.33 \\
(1.37)\end{array}$ & $\begin{array}{l}2.55 \\
(1.40)\end{array}$ & $\begin{array}{l}17.06 \\
(6.34)\end{array}$ & \\
\hline $45+$ years & 110 & $\begin{array}{l}3.30 \\
(1.44)\end{array}$ & $\begin{array}{l}2.20 \\
(1.34)\end{array}$ & $\begin{array}{l}2.55 \\
(1.41)\end{array}$ & $\begin{array}{l}2.26 \\
(1.27)\end{array}$ & $\begin{array}{l}3.23 \\
(1.46)\end{array}$ & $\begin{array}{l}2.45 \\
(1.35)\end{array}$ & $\begin{array}{l}15.99 \\
(6.15)\end{array}$ & \\
\hline Total N & 1603 & $\begin{array}{l}3.08 \\
(1.52)\end{array}$ & $\begin{array}{l}2.73 \\
(1.73)\end{array}$ & $\begin{array}{l}3.28 \\
(1.53)\end{array}$ & $\begin{array}{l}2.78 \\
(1.47)\end{array}$ & $\begin{array}{l}3.01 \\
(1.63)\end{array}$ & $\begin{array}{l}2.64 \\
(1.53)\end{array}$ & $\begin{array}{l}17.52 \\
(6.56)\end{array}$ & \\
\hline
\end{tabular}

a The mean difference is significant at the 0.05 level

b Adjustment for multiple comparisons: Bonferroni

${ }^{1}$ Compared to the $12-18$ age group

${ }^{2}$ Compared to the 19-24 age group

${ }^{3}$ Compared to the 25-34 age group

${ }^{4}$ Compared to the 35-44 age group

${ }^{5}$ Compared to the $45+$ age group 


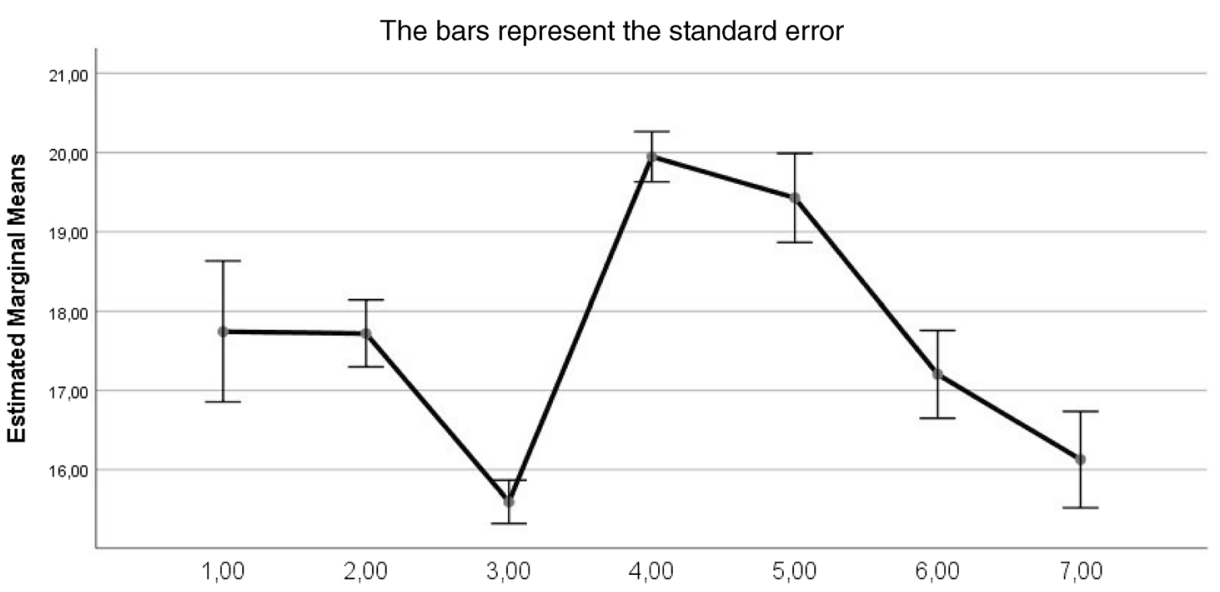

Fig. 3 SABAS total score variation by Kail and Cavanaugh's age groups

adults are unknown. Nevertheless, it appears that this older age group relies on smartphone use primarily for maintaining contact with family and friends. Smartphone use for communication purposes does not appear to cause any negative consequences for older adults, suggesting that these individuals are more motivated to maintain good quality social connections (Chan 2018). The findings of the present study also showed that specific addiction components of smartphone usage were more dominant at different age groups. Consequently, smartphone-related addictive components show different patterns for diverse age groups.

The analyses based on Armstrong's age groups showed that the 3-11-year old age group manifested significantly higher salience than the 12-19-year old age group, but lower than the 20-34-year old age group. This latter group showed higher salience than the 12-19, 35-50, and the 50+ year old age groups on this addiction component. In other words, salience was the highest among the 20-34-year old age group, showing that smartphone use is one of the most important activities in their lives. Given that social life, travel, and job-seeking is relatively typical in this age group, the highest level of salience may not necessary reflect addiction, although it clearly shows that this age group is highly dependent on their smartphone (Long et al. 2016). These results

Table 5 Tests of between-participant effects and significance of the problematic use components by Kail and Cavanaugh's age groups

\begin{tabular}{llllllll}
\hline Source & Dependent variable & $\begin{array}{l}\text { Type III sum } \\
\text { of square }\end{array}$ & df. & $\begin{array}{l}\text { Mean } \\
\text { square }\end{array}$ & $F$ & Sig.* & $\begin{array}{l}\text { Partial Eta } \\
\text { squared }\end{array}$ \\
\hline Age group & Salience & 361.56 & 6 & 60.26 & 28.71 & $p<0.001$ & 0.097 \\
& Conflict & 368.86 & 6 & 61.47 & 22.02 & $p<0.001$ & 0.076 \\
& Mood modification & 149.55 & 6 & 24.92 & 11.11 & $p<0.001$ & 0.040 \\
& Tolerance & 112.14 & 6 & 18.69 & 8.89 & $p<0.001$ & 0.032 \\
& Withdrawal symptoms & 725.44 & 6 & 120.90 & 54.59 & $p<0.001$ & 0.170 \\
& Relapse & 254.40 & 6 & 42.40 & 19.31 & $p<0.001$ & 0.068 \\
& SABAS total score & 5043.69 & 6 & 840.61 & 20.96 & $p<0.001$ & 0.073 \\
\hline
\end{tabular}

*Computed using alpha $=0.05$ 
Table 6 Multiple comparison results for the problematic use components by Kail and Cavanaugh's age groups

\begin{tabular}{|c|c|c|c|c|c|}
\hline \multirow{2}{*}{$\begin{array}{l}\text { Dependent variable } \\
\text { Salience }\end{array}$} & \multicolumn{2}{|c|}{ Age group (years) } & \multirow{2}{*}{$\frac{\text { Mean difference }(\mathrm{I}-\mathrm{J})}{0.862^{*}}$} & \multirow{2}{*}{$\begin{array}{l}\text { Std. error } \\
0.224\end{array}$} & \multirow{2}{*}{$\frac{\text { Sig. }^{\mathrm{b}}}{0.003}$} \\
\hline & $3-6$ & $7-11$ & & & \\
\hline & & $12-18$ & $1.401^{*}$ & 0.212 & $p<0.001$ \\
\hline & & $19-24$ & 0.329 & 0.216 & 1.000 \\
\hline & & $25-34$ & 0.286 & 0.241 & 1.000 \\
\hline & & $35-44$ & 0.533 & 0.240 & 0.557 \\
\hline & & $45+$ & 0.567 & 0.247 & 0.457 \\
\hline & $7-11$ & $12-18$ & $0.538^{*}$ & 0.114 & $p<0.001$ \\
\hline & & $19-24$ & $-0.533^{*}$ & 0.121 & $p<0.001$ \\
\hline & & $25-34$ & $-0.576^{*}$ & 0.161 & 0.008 \\
\hline & & $35-44$ & -0.329 & 0.160 & 0.836 \\
\hline & & $45+$ & -0.296 & 0.169 & 1.000 \\
\hline & $12-18$ & $19-24$ & $-1.071^{*}$ & 0.096 & $p<0.001$ \\
\hline & & $25-34$ & $-1.114^{*}$ & 0.144 & $p<0.001$ \\
\hline & & $35-44$ & $-0.867^{*}$ & 0.142 & $p<0.001$ \\
\hline & & $45+$ & $-0.834^{*}$ & 0.153 & $p<0.001$ \\
\hline & $19-24$ & $25-34$ & -0.043 & 0.148 & 1.000 \\
\hline & & $35-44$ & 0.204 & 0.146 & 1.000 \\
\hline & & $45+$ & 0.237 & 0.156 & 1.000 \\
\hline & $25-34$ & $35-44$ & 0.247 & 0.180 & 1.000 \\
\hline & & $45+$ & 0.280 & 0.189 & 1.000 \\
\hline & $35-44$ & $45+$ & 0.033 & 0.187 & 1.000 \\
\hline \multirow[t]{21}{*}{ Conflict } & $3-6$ & $7-11$ & $1.354^{*}$ & 0.259 & $p<0.001$ \\
\hline & & $12-18$ & $1.487^{*}$ & 0.245 & $p<0.001$ \\
\hline & & $19-24$ & 0.455 & 0.249 & 1.000 \\
\hline & & $25-34$ & 0.694 & 0.278 & 0.266 \\
\hline & & $35-44$ & $1.143^{*}$ & 0.277 & 0.001 \\
\hline & & $45+$ & $1.579^{*}$ & 0.285 & $p<0.001$ \\
\hline & $7-11$ & $12-18$ & 0.133 & 0.131 & 1.000 \\
\hline & & $19-24$ & $-0.899^{*}$ & 0.139 & $p<0.001$ \\
\hline & & $25-34$ & $-0.660^{*}$ & 0.186 & 0.008 \\
\hline & & $35-44$ & -0.211 & 0.184 & 1.000 \\
\hline & & $45+$ & 0.225 & 0.195 & 1.000 \\
\hline & $12-18$ & $19-24$ & $-1.032^{*}$ & 0.111 & $p<0.001$ \\
\hline & & $25-34$ & $-0.793^{*}$ & 0.166 & $p<0.001$ \\
\hline & & $35-44$ & -0.344 & 0.164 & 0.749 \\
\hline & & $45+$ & 0.092 & 0.176 & 1.000 \\
\hline & $19-24$ & $25-34$ & 0.239 & 0.170 & 1.000 \\
\hline & & $35-44$ & $0.688^{*}$ & 0.168 & 0.001 \\
\hline & & $45+$ & $1.124^{*}$ & 0.180 & $p<0.001$ \\
\hline & $25-34$ & $35-44$ & 0.449 & 0.208 & 0.649 \\
\hline & & $45+$ & $0.885^{*}$ & 0.218 & 0.001 \\
\hline & $35-44$ & $45+$ & 0.436 & 0.216 & 0.911 \\
\hline \multirow[t]{13}{*}{ Mood modification } & $3-6$ & $7-11$ & 0.000 & 0.232 & 1.000 \\
\hline & & $12-18$ & 0.458 & 0.219 & 0.774 \\
\hline & & $19-24$ & 0.551 & 0.223 & 0.289 \\
\hline & & $25-34$ & 0.496 & 0.249 & 0.978 \\
\hline & & $35-44$ & $0.975^{*}$ & 0.248 & 0.002 \\
\hline & & $45+$ & $1.193^{*}$ & 0.255 & $p<0.001$ \\
\hline & $7-11$ & $12-18$ & $0.458^{*}$ & 0.118 & 0.002 \\
\hline & & $19-24$ & $0.551^{*}$ & 0.125 & $p<0.001$ \\
\hline & & $25-34$ & 0.496 & 0.167 & 0.062 \\
\hline & & $35-44$ & $0.975^{*}$ & 0.165 & $p<0.001$ \\
\hline & & $45+$ & $1.193^{*}$ & 0.175 & $p<0.001$ \\
\hline & $12-18$ & $19-24$ & 0.093 & 0.099 & 1.000 \\
\hline & & $25-34$ & 0.038 & 0.148 & 1.000 \\
\hline
\end{tabular}


Table 6 (continued)

\begin{tabular}{|c|c|c|c|c|c|}
\hline Dependent variable & \multicolumn{2}{|c|}{ Age group (years) } & \multirow{2}{*}{$\frac{\text { Mean difference (I-J) }}{0.517^{*}}$} & \multirow{2}{*}{$\frac{\text { Std. error }}{0.147}$} & \multirow{2}{*}{$\frac{\text { Sig. }{ }^{\mathrm{b}}}{0.009}$} \\
\hline & & $35-44$ & & & \\
\hline & & $45+$ & $0.735^{*}$ & 0.158 & $p<0.001$ \\
\hline & 19-24 & $25-34$ & -0.055 & 0.153 & 1.000 \\
\hline & & $35-44$ & 0.424 & 0.151 & 0.102 \\
\hline & & $45+$ & $0.642^{*}$ & 0.161 & 0.002 \\
\hline & $25-34$ & $35-44$ & 0.479 & 0.186 & 0.214 \\
\hline & & $45+$ & $0.697^{*}$ & 0.195 & 0.008 \\
\hline & $35-44$ & $45+$ & 0.218 & 0.193 & 1.000 \\
\hline \multirow[t]{21}{*}{ Tolerance } & $3-6$ & $7-11$ & $-0.859^{*}$ & 0.225 & 0.003 \\
\hline & & $12-18$ & -0.432 & 0.212 & 0.884 \\
\hline & & $19-24$ & -0.310 & 0.216 & 1.000 \\
\hline & & $25-34$ & -0.136 & 0.241 & 1.000 \\
\hline & & $35-44$ & -0.038 & 0.240 & 1.000 \\
\hline & & $45+$ & 0.145 & 0.247 & 1.000 \\
\hline & $7-11$ & $12-18$ & $0.428^{*}$ & 0.114 & 0.004 \\
\hline & & $19-24$ & $0.549^{*}$ & 0.121 & $p<0.001$ \\
\hline & & $25-34$ & $0.723^{*}$ & 0.161 & $p<0.001$ \\
\hline & & $35-44$ & $0.821^{*}$ & 0.160 & $p<0.001$ \\
\hline & & $45+$ & $1.004^{*}$ & 0.170 & $p<0.001$ \\
\hline & $12-18$ & $19-24$ & 0.121 & 0.096 & 1.000 \\
\hline & & $25-34$ & 0.295 & 0.144 & 0.839 \\
\hline & & $35-44$ & 0.393 & 0.142 & 0.118 \\
\hline & & $45+$ & $0.577^{*}$ & 0.153 & 0.003 \\
\hline & 19-24 & $25-34$ & 0.174 & 0.148 & 1.000 \\
\hline & & $35-44$ & 0.272 & 0.146 & 1.000 \\
\hline & & $45+$ & 0.455 & 0.156 & 0.076 \\
\hline & $25-34$ & $35-44$ & 0.098 & 0.180 & 1.000 \\
\hline & & $45+$ & 0.281 & 0.189 & 1.000 \\
\hline & $35-44$ & $45+$ & 0.183 & 0.187 & 1.000 \\
\hline \multirow[t]{21}{*}{ Withdrawal symptoms } & $3-6$ & $7-11$ & -0.438 & 0.231 & 1.000 \\
\hline & & $12-18$ & -0.087 & 0.218 & 1.000 \\
\hline & & $19-24$ & $-1.693^{*}$ & 0.222 & $p<0.001$ \\
\hline & & $25-34$ & $-1.537^{*}$ & 0.248 & $p<0.001$ \\
\hline & & $35-44$ & $-1.134^{*}$ & 0.247 & $p<0.001$ \\
\hline & & $45+$ & $-1.035^{*}$ & 0.253 & 0.001 \\
\hline & $7-11$ & $12-18$ & 0.350 & 0.117 & 0.059 \\
\hline & & $19-24$ & $-1.255^{*}$ & 0.124 & $p<0.001$ \\
\hline & & $25-34$ & $-1.099^{*}$ & 0.166 & $p<0.001$ \\
\hline & & $35-44$ & $-0.696^{*}$ & 0.164 & $p<0.001$ \\
\hline & & $45+$ & $-0.598^{*}$ & 0.174 & 0.013 \\
\hline & $12-18$ & $19-24$ & $-1.606^{*}$ & 0.099 & $p<0.001$ \\
\hline & & $25-34$ & $-1.450^{*}$ & 0.147 & $p<0.001$ \\
\hline & & $35-44$ & $-1.047^{*}$ & 0.146 & $p<0.001$ \\
\hline & & $45+$ & $-0.948^{*}$ & 0.157 & $p<0.001$ \\
\hline & 19-24 & $25-34$ & 0.156 & 0.152 & 1.000 \\
\hline & & $35-44$ & $0.559^{*}$ & 0.150 & 0.004 \\
\hline & & $45+$ & $0.658^{*}$ & 0.160 & 0.001 \\
\hline & $25-34$ & $35-44$ & 0.403 & 0.185 & 0.620 \\
\hline & & $45+$ & 0.501 & 0.194 & 0.206 \\
\hline & $35-44$ & $45+$ & 0.098 & 0.192 & 1.000 \\
\hline \multirow{5}{*}{ Relapse } & $3-6$ & $7-11$ & $-0.895^{*}$ & 0.230 & 0.002 \\
\hline & & $12-18$ & $-0.679^{*}$ & 0.217 & 0.037 \\
\hline & & $19-24$ & $-1.537^{*}$ & 0.221 & $p<0.001$ \\
\hline & & $25-34$ & $-1.489^{*}$ & 0.247 & $p<0.001$ \\
\hline & & $35-44$ & $-0.940^{*}$ & 0.246 & 0.003 \\
\hline
\end{tabular}


Table 6 (continued)

\begin{tabular}{|c|c|c|c|c|c|}
\hline Dependent variable & \multicolumn{2}{|c|}{ Age group (years) } & Mean difference (I-J) & Std. error & \multirow[t]{2}{*}{ Sig. ${ }^{b}$} \\
\hline & & $45+$ & $-0.832^{*}$ & 0.252 & \\
\hline & $7-11$ & $12-18$ & 0.216 & 0.117 & 1.000 \\
\hline & & $19-24$ & $-0.642^{*}$ & 0.124 & $p<0.001$ \\
\hline & & $25-34$ & $-0.594^{*}$ & 0.165 & 0.007 \\
\hline & & $35-44$ & -0.044 & 0.163 & 1.000 \\
\hline & & $45+$ & 0.063 & 0.173 & 1.000 \\
\hline & $12-18$ & $19-24$ & $-0.858^{*}$ & 0.098 & $p<0.001$ \\
\hline & & $25-34$ & $-0.810^{*}$ & 0.147 & $p<0.001$ \\
\hline & & $35-44$ & -0.261 & 0.145 & 1.000 \\
\hline & & $45+$ & -0.153 & 0.156 & 1.000 \\
\hline & $19-24$ & $25-34$ & 0.048 & 0.151 & 1.000 \\
\hline & & $35-44$ & $0.597^{*}$ & 0.149 & 0.001 \\
\hline & & $45+$ & $0.705^{*}$ & 0.160 & $p<0.001$ \\
\hline & $25-34$ & $35-44$ & 0.549 & 0.184 & 0.061 \\
\hline & & $45+$ & $0.657^{*}$ & 0.193 & 0.014 \\
\hline & $35-44$ & $45+$ & 0.107 & 0.191 & 1.000 \\
\hline
\end{tabular}

*The mean difference is significant at the 0.05 level

${ }^{\mathrm{b}}$ Adjustment for multiple comparisons: Bonferroni

concur with Lopez-Fernandez et al. (2017) who showed that in the 19-24-year old age group, the most frequent activity was social networking (Lopez-Fernandez et al. 2017).

While the conflict component in the 20-34-year old age group was lower than in 3-11 and in 12-19-year old age groups, this component was significantly higher than in the two older age groups. These results may reflect diminishing parental control while showing that partners, employers, and/or friends may confront individuals in this age group when noticing the individual's exaggerated use of their smartphone. These findings are in concordance with the results of other studies reporting that positive parental control can prevent excessive smartphone use (Hwang et al. 2017), while the lack of parental involvement in setting rules for the amount and content of smartphone use may be a risk factor for problematic smartphone use among children (Lian et al. 2016).

Mood modification was the highest in the 3-11 age groups and after that decreased continuously, with significant scores in the following age groups. The lowest average scores were in the group of over 50-year olds. Such results may be attributable to increasing age and the maturation processes, as well as the psychological factors, such as an increased selfcontrol. These results also concur with Kim et al. (2016) who found self-control was negatively correlated with problematic smartphone use (Kim et al. 2016).

The level of self-control ability may serve as a possible explanation of why the lower age groups spent increasing amounts of time with their smartphone. The 3-11-year-olds had significantly higher scores for the tolerance component than the 12-19, 20-34, 35-50, and the 50+-year-old respondents, and is in line with other studies who found participants who spent more time on social network sites reported higher addictive tendencies (Wu et al. 2013). At the same time, duration of smartphone use is positively correlated with smartphone addiction (Gökçearslan et al. 2016).

When respondents cannot use their smartphone, they experience negative feelings, leading to withdrawal symptoms and fear of missing out (Eide et al. 2018; Stieger and Lewetz 2018). In the present study, 20-34-year-olds reported this component the most, but the results also showed that 
withdrawal occurred among the 35-50 and 50+ age groups. Older-age individuals appear to display deeper physical and psychological symptoms associated with withdrawal than the younger age groups. According to other research, in a sample of 18-68-year olds (mean age = 30.96), age was a negative predictor of problematic smartphone use (Mitchell and Hussain 2018).

Relapse showed the highest scores in the 20-34 age group and was significantly lower than in the 35-50 age group. This tendency appeared in the 50+ years age group too. Lower scores were observed in the younger age groups, especially compared to the 3-11-year olds. However, there appears to be no relevant previous research on the relapse component of problematic smartphone use. The analyses based on Kail and Cavanaugh's age group classification showed that the typical activities of the respondents can determine the amount of smartphone use. According to both analyses, the young adult age group reported the highest scores and adolescents the lowest. The preschoolers' (3-6 years) scores on salience was the highest among the age groups within the new analysis, followed by the average scores of 25-34-year and 1924-year groups. The results of the kindergarten children differed significantly from the 7-11and 12-18-year groups; therefore, the scores of the latter two age groups differed significantly from those of both the younger and older age groups, in terms of salience.

Conflict showed higher scores among 3-6-year-olds, as well as in the 19-24 and 2534 year groups. According to the results, problematic smartphone use among preschool children is likely to be characterized by conflicts with parents, while in the case of a young adult it may lead to relationship or work conflicts. These findings suggest, in line with other research, that educational or occupational stress can predict problematic smartphone use and dependence (Lopez-Fernandez et al. 2018).

Mood modification showed the same tendency, with younger age groups having the highest scores, with decreasing scores with increasing age. The results of the 7-11-year-olds were significantly different from those aged between 12 and 18 and 19-24 years, who had a decreasing tendency. The lowest average scores were among those aged over 45 years.

The average scores for the 7-11-year olds were the highest for tolerance and decreased with age. Furthermore, it is evident that their scores differed significantly from all other age groups. This age group might have the greatest interest in new information and new tools and comes at a time when their learning ability is supposed to be the highest (Meadows 2017).

According to the results, withdrawal symptoms were the highest among the 19-24-year olds and those aged between 25 and 34 years. The lowest scores were among preschooler where the lack of a smartphone manifests more in mood modification than in withdrawal. The same trend was observed in the relapse component, which shows the highest scores among those aged 1924 years old and 25-34 years and lowest among the 3-6-year old age group. The scores of those aged 3-6 years were significantly different from the scores of all other age groups.

There are several differences between the results of the two age group classifications. Regarding salience, the Armstrong analysis score (3-11 years = 3.18) was influenced by the high score of the kindergarten age group, compared to the scores of those aged 7-11 years. The results for the next age group (12-19/20 years) were almost the same for both age group classifications. Kail and Cavanaugh distributed Armstrong's third group (20-34 years) into two age bands (1924 years and 25-34 years), yet the salience scores remained the same. The results of the final two age bands were similar in both distributions. Concerning the conflict component, the second analysis provided significant clarifications because higher scores tended to be among 3-6-year olds, and then decreased significantly. Younger adults (19-24 years) had higher scores than among the 19/20-34-year old. Mood modification in the 3-11-year age group had the highest scores, and the second analysis with the data split did not change the findings. The high scores of 
the 7-11 age group, compared to the second analysis, caused the high scores of tolerance in the first analysis. The presence of withdrawal symptoms in the 3-6-year-old group were the lowest, and highest among those aged 19-24 years. This decreased among older age groups but remained higher than in any age group between 3 and 19 years. Relapse scores showed a similar distribution across the age groups. Overall, the Kail and Cavanaugh age group classifications were better at detecting age characteristics and the most dominant addiction components by age.

\section{Limitations}

The most major limitations in the present study were the use of self-reported data (which are subject to well-known biases) and self-selected samples (so the present findings need replicating with more representative samples). The nature of cross-sectional convenience samples is a common limitation in studies such as this and nothing can be concluded regarding causal factors. Furthermore, some age groups, such as the kindergarten sample, were small which may have influenced the findings.

\section{Conclusion}

The SABAS, based on 'components model of addiction', has proven to be a reliable tool to assess problematic smartphone use across different age groups. Problematic smartphone use appears to have specific patterns according to age, with dominance of salience, mood modification and conflict in childhood, and tolerance, withdrawal symptoms, and relapse in adulthood. Some age groups appear to be at greater risk for problematic smartphone use, so prevention should focus on these ages. In addition, targeted prevention can be used to maximum efficiency depending on the characteristics of the age group using information concerning the dominant components of problematic smartphone use at specific age levels.

Funding The study was supported by the Hungarian National Research, Development and Innovation Office (KKP126835).

\section{Compliance with Ethical Standards}

Conflict of Interest The authors declare that they do not have any interests that could constitute a real, potential, or apparent conflict of interest with respect to their involvement in the publication. The authors also declare that they do not have any financial or other relations (e.g., directorship, consultancy, or speaker fee) with companies, trade associations, unions, or groups (including civic associations and public interest groups) that may gain or lose financially from the results or conclusions in the study. Sources of funding are acknowledged.

Ethical Approval All procedures performed in this study involving human participants were in accordance with the ethical standards of University's Research Ethics Board and with the 1975 Helsinki Declaration.

Informed Consent Informed consent was obtained from all participants.

Open Access This article is distributed under the terms of the Creative Commons Attribution 4.0 International License (http:/creativecommons.org/licenses/by/4.0/), which permits unrestricted use, distribution, and reproduction in any medium, provided you give appropriate credit to the original author(s) and the source, provide a link to the Creative Commons license, and indicate if changes were made. 


\section{References}

Andreassen, C. S., Griffiths, M. D., Hetland, J., \& Pallesen, S. (2012). Development of a work addiction scale. Scandinavian Journal of Psychology, 53(3), 265-272.

Andreassen, C. S., Griffiths, M. D., Pallesen, S., Bilder, R. M., Torsheim, T., \& Aboujaoude, E. (2015). The Bergen Shopping Addiction Scale: Reliability and validity of a brief screening test. Frontiers in Psychology, $6,1374$.

Andreassen, C. S., Billieux, J., Griffiths, M. D., Kuss, D. J., Demetrovics, Z., Mazzoni, E., \& Pallesen, S. (2016). The relationship between addictive use of social media and video games and symptoms of psychiatric disorders: A large-scale cross-sectional study. Psychology of Addictive Behaviors, 30(2), 252-262.

Armstrong, T. (2007). The human odyssey: Navigating the twelve stages of life. New York: Sterling Publishing Company.

Berger, S., Wyss, A. M., \& Knoch, D. (2018). Low self-control capacity is associated with immediate responses to smartphone signals. Computers in Human Behavior, 86, 45-51.

Bőthe, B., Tóth-Király, I., Zsila, Á., Griffiths, M. D., Demetrovics, Z., \& Orosz, G. (2018). The development of the Problematic Pornography Consumption Scale (PPCS). Journal of Sex Research, 55(3), 395-406.

Chan, M. (2018). Mobile-mediated multimodal communications, relationship quality and subjective well-being: An analysis of smartphone use from a life course perspective. Computers in Human Behavior, 87, 254-262.

Csibi, S., Demetrovics, Z., \& Szabo, A. (2016). Development and psychometric validation of the Brief Smartphone Addiction Scale (BASS) with schoolchildren. Psychiatria Hungarica: A Magyar Pszichiatriai Tarsasag Tudomanyos Folyoirata, 31(1), 71-77.

Csibi, S., Griffiths, M. D., Cook, B., Demetrovics, Z., \& Szabo, A. (2018). The psychometric properties of the Smartphone Application-Based Addiction Scale (SABAS). International Journal of Mental Health and Addiction, 16(2), 393-403.

Csibi, S., Demetrovics, Z., \& Szabo, A. (2019). Validation of the Brief Addiction to Smartphone Scale (BASS) and the Hungarian Smartphone Deprivation Inventory (HSDI) in adult sample. Psychiatria Hungarica, 34(1), in press.

Csibi, S., Demetrovics, Z., \& Szabo, A. (2017). Validation of Hungarian Smartphone Deprivation Inventory (HSDI) with school children. Psychiatria Hungarica, 32(3), 307-312.

de-Sola, J., Talledo, H., de Fonseca, F. R., \& Rubio, G. (2017). Prevalence of problematic cell phone use in an adult population in Spain as assessed by the Mobile Phone Problem Use Scale (MPPUS). PLoS One, 12(8), e0181184.

Eide, T. A., Aarestad, S. H., Pallesen, S., Andreassen, C. S., \& Bilder, R. M. (2018). Smartphone restriction and its effect on subjective withdrawal related scores. Frontiers in Psychology, 9, 1444.

Fullwood, C., Quinn, S., Kaye, L. K., \& Redding, C. (2017). My virtual friend: A qualitative analysis of the attitudes and experiences of smartphone users: Implications for smartphone attachment. Computers in Human Behavior, 75, 347-355.

Gökçearslan, Ș., Mumcu, F. K., Haşlaman, T., \& Çevik, Y. D. (2016). Modelling smartphone addiction: The role of smartphone usage, self-regulation, general self-efficacy and cyberloafing in university students. Computers in Human Behavior, 63, 639-649.

Griffiths, M.D (2005). A components model of addiction within a biopsychosocial framework. Journal of Substance Use, 10, 191-197

Hwang, Y., Choi, I., Yum, J. Y., \& Jeong, S. H. (2017). Parental mediation regarding children's smartphone use: Role of protection motivation and parenting style. Cyberpsychology, Behavior and Social Networking, 20(6), 362-368.

Ihm, J. (2018). Social implications of children's smartphone addiction: The role of support networks and social engagement. Journal of Behavioral Addictions, 7(2), 473-481.

Kail, R. V., \& Cavanaugh, J. C. (2018). Human development: A life-span view. Boston: Cengage Learning.

Kim, J. K., \& Kang, Y. S. (2016). The effects of young children's smartphone use experience on their parents perceptions and needs and their selfregulation. International Journal of Applied Engineering Research, 11(2), 1208-1211.

Kim, Y., Jeong, J. E., Cho, H., Jung, D. J., Kwak, M., Rho, M. J., et al. (2016). Personality factors predicting smartphone addiction predisposition: Behavioral inhibition and activation systems, impulsivity, and selfcontrol. PLoS One, 11(8), e0159788.

Kuss, D. J., Kanjo, E., Crook-Rumsey, M., Kibowski, F., Wang, G. Y., \& Sumich, A. (2018). Problematic mobile phone use and addiction across generations: The roles of psychopathological symptoms and smartphone use. Journal of Technology in Behavioral Science, 3(3), 141-149.

Kwon, M. S., Yoon, O. S., Noh, G. Y., Chun, J., \& Han, S. (2017). Smartphone addiction level and smartphone use expectation in adults. International Information Institute (Tokyo). Information, 20(8B), 6003-6010. 
Lane, W., \& Manner, C. (2011). The impact of personality traits on smartphone ownership and use. International Journal of Business and Social Science, 2(17), 22-28.

Lemola, S., Perkinson-Gloor, N., Brand, S., Dewald-Kaufmann, J. F., \& Grob, A. (2015). Adolescents' electronic media use at night, sleep disturbance, and depressive symptoms in the smartphone age. Journal of Youth and Adolescence, 44(2), 405-418.

Lian, L., You, X., Huang, J., \& Yang, R. (2016). Who overuses smartphones? Roles of virtues and parenting style in smartphone addiction among Chinese college students. Computers in Human Behavior, 65, 92-99.

Long, J., Liu, T.-Q., Liao, Y.-H., Qi, C., He, H.-Y., Chen, S.-B., \& Billieux, J. (2016). Prevalence and correlates of problematic smartphone use in a large random sample of Chinese undergraduates. BMC Psychiatry, 16, 408.

Lopez-Fernandez, O., Kuss, D. J., Romo, L., Morvan, Y., Kern, L., Graziani, P., et al. (2017). Self-reported dependence on mobile phones in young adults: A European cross-cultural empirical survey. Journal of Behavioral Addictions, 6(2), 168-177.

Lopez-Fernandez, O., Männikkö, N., Kääriäinen, M., Griffiths, M. D., \& Kuss, D. J. (2018). Mobile gaming and problematic smartphone use: A comparative study between Belgium and Finland. Journal of Behavioral Addictions, 7(1), 88-99.

Meadows, S. (2017). Developing thinking: Approaches to children's cognitive development. London: Routledge.

Mitchell, L., \& Hussain, Z. (2018). Predictors of problematic smartphone use: An examination of the Integrative Pathways Model and the role of age, gender, impulsiveness, excessive reassurance seeking, extraversion, and depression. Behavioral Sciences, 8(8), 74-87.

Ofcom. (2017). Children and parents: Media use and attitudes report. London: The Office of Communications.

Park, C., \& Park, Y. R. (2014). The conceptual model on smart phone addiction among early childhood. International Journal of Social Science and Humanity, 4(2), 147-150.

Stieger, S., \& Lewetz, D. (2018). A week without using social media: results from an ecological momentary intervention study using smartphones. Cyberpsychology, Behavior and Social Networking, 21(10), 618-624.

Terry, A., Szabo, A., \& Griffiths, M. D. (2004). The exercise addiction inventory: A new brief screening tool. Addiction Research and Theory, 12, 489-499.

Van Deursen, A. J., Bolle, C. L., Hegner, S. M., \& Kommers, P. A. (2015). Modeling habitual and addictive smartphone behavior: The role of smartphone usage types, emotional intelligence, social stress, selfregulation, age, and gender. Computers in Human Behavior, 45, 411-420.

Wu, A. M., Cheung, V. I., Ku, L., \& Hung, E. P. (2013). Psychological risk factors of addiction to social networking sites among Chinese smartphone users. Journal of Behavioral Addictions, 2(3), 160-166.

Yadav, S., \& Chakraborty, P. (2017). Children aged two to four are able to scribble and draw using a smartphone app. Acta Paediatrica, 106(6), 991-994.

Publisher's Note Springer Nature remains neutral with regard to jurisdictional claims in published maps and institutional affiliations. 